\title{
Study on Quantification Method for Dispersion and Distribution of Sphere/-like Particles and Relationship with AC/DC Breakdown Strength in Polymer Nanocomposites
}

\author{
Dayuan Qiang, Y. Wang, F. N. Alhabill, G. Chen and T. Andritsch \\ University of Southampton, \\ Department of Electronics and Computer Science, \\ Highfield, Southampton, \\ SO17 1BJ, U.K
}

\begin{abstract}
Polymer nanocomposites are more and more researched and employed as dielectrics in electrical power equipment. However, the bad dispersion and distribution of particles are often reported to deteriorate the dielectric properties of polymer nanocomposites. In most cases, researchers tend to use SEM/TEM images with imprecise descriptions to describe these two factors, and there was still no clear relationship between their quantified particle dispersion characteristics and dielectric properties of polymer nanocomposites. This work is to, first, propose a combined quantification method to estimate the dispersion and distribution of spherical/ellipsoidal particles/aggregates in polymer nanocomposites based on $\mathrm{SEM}$ images of epoxy $\mathrm{SiO}_{2}$ nanocomposites. Based on the proposed quantification method, epoxy nanocomposite with surface treated $\mathrm{SiO}_{2}$ shows overall better dispersion and distribution of particles/aggregates than those with untreated ones. The presence of agglomerations would lead to the enhancement of mobility of charge carriers and thus reduction of breakdown strength, which become more obvious with the growth of filler loadings. It is found that, in AC breakdown tests, dispersion and distribution of particles/aggregates show little influence on the reduction of AC breakdown strength. However, those should be the main factor which influences the DC breakdown strength in epoxy nanocomposites with a variation of filler loading concentrations.
\end{abstract}

Index Terms - Nanotechnology, dielectric breakdown, silica, epoxy resins, electron microscopy, quantification method

\section{INTRODUCTION}

NANOCOMPOSITES initially started from areas in ceramics as inorganic and metal powder fillers [1]. Because of broader usage demand on materials and specific properties of nanocomposites, such as thermal, electrical and mechanical ones, they nowadays have been introduced into not only our daily life but also in high voltage and power engineering [2]. Many researchers have introduced the nano-materials as particles into polymers, such as PE and epoxy resins, and wish to modify/enhance their properties as insulators. Due to the extremely large surface area of nano-sized particles, even in a small loading concentration, the presence of particles has been

Manuscript received on xx Month 20yy, in final form xx Month 20yy, accepted xx Month 20yy. Corresponding author: D. Qiang. widely reported to influence the characteristics of polymers and further may affect the dielectric properties of the resultant composites [3]. For example, the interfacial characteristics between nano-particles and base polymer materials play an important role in determining the electrical properties due to the presence of such large surface area [4]. However, the presence of nano-sized fillers shows no or significant improvement in dielectric breakdown (BD) behavior of epoxy resin composites. Similar results were also observed by Imai et al. (2006) [5], Singha and Thomas (2008) [6]. The addition of nano-particles to epoxy resin does however significantly reduce the shape parameter $(\beta)$, a result which is contrary to several other studies, including Singha et al. and Nelson et al. [7]. In addition, Wang et al. found the surface treatment could modify the reduction of $\mathrm{AC}$ breakdown strength and showed a higher value than pure samples in some low filler loading concentrations but still worse in high loadings [8]. He attributes this to the distribution of 
particles with relatively simple quantitative data. Moreover, it also found that surface treatment could help enhance the breakdown strength of nanocomposites compared to the untreated ones [9].

As morphology of mixing state of particles in polymers is reported to affect the insulating properties of polymer nanocomposites and worse one may result in poor performance, many researchers have sought to achieve good mixing states by different particle surface treatment methods [10] and further characterise the polymer nanocomposites quantitatively $[11,12]$. Kim et al. introduced two aspects to figure out the morphology of mixing state of particles [11]. The first is the dispersion of particles, which is related to the size reduction of the agglomerations of particles. The second is the distribution of particles/aggregates (refer to tightly/loosely agglomerated particles), which involves the distribution state of particles/aggregates in matrix of polymers. Hui et al. then quantify the mixing in nanocomposites with these two aspects via analysing the TEM images of XLPE nanocomposites [12]. However, there is few research on quantification of SEM images, which is also a widely used characterization method of mixing morphology, and most analyses were usually based on SEM images with imprecise descriptions. Moreover, in most of the time, researchers tend to use imprecise descriptions to describe these two factors through SEM/TEM images, although some researches considered the quantitative methods [13], there was still no direct relationship between their quantified morphology characteristics and dielectric properties of polymer nanocomposites in different filler loadings.

Thus, the main objectives in this paper are first to propose a quantification method to estimate the dispersion and distribution of particles/aggregates in polymer nanocomposites based on SEM images of epoxy $\mathrm{SiO}_{2}$ (treated and untreated ones) nanocomposites. Second, it is to use the quantitatively characterised morphology to find out how nano-scaled fillers influence dielectric breakdown of epoxy nanocomposites, to investigate the effect of interfacial regions between the matrix and nano-fillers on the insulating performance.

\section{EXPERIMENTAL}

\subsection{MATERIALS}

The samples were prepared by using bisphenol-A diglycidyl ether (D.E.R. 332, density $1.16 \mathrm{~g} \cdot \mathrm{cm}^{-3}$ ) cured with polyether amine hardener (Jeffamine D-230, density 0.948 $\mathrm{g} \cdot \mathrm{cm}^{-3}$ ) supplied by Huntsman. The fillers used in the study are commercially available untreated $\mathrm{SiO}_{2}$ fillers provided by Sigma-Aldrich. The average particle size (APS) based on BET measuring method is $10 \sim 20 \mathrm{~nm}$. Moreover, trimethoxy (octyl) silane is used as a coupling agent to produce treated nano- $\mathrm{SiO}_{2}$ (same preparation method was introduced in [14] and samples are coded as $\mathrm{C} 8$ treated nano- $\mathrm{SiO}_{2}$ ) in order to eliminate the hydroxyl groups on the surface of $\mathrm{SiO}_{2}$ particles and achieve better dispersion.

\subsection{SAMPLE PREPARATION}

The epoxy nanocomposites samples with different loading concentrations of nano-fillers (untreated $\mathrm{SiO}_{2}$ and $\mathrm{C} 8$ treated
$\left.\mathrm{SiO}_{2}\right)$ in $0.5 \mathrm{wt} \%, 1 \mathrm{wt} \%, 3 \mathrm{wt} \%$ and $5 \mathrm{wt} \%$ were prepared. Before preparation, in order to reduce its viscosity, the epoxy resin is pre-heated to $50{ }^{\circ} \mathrm{C}$ in the oven $(1 \mathrm{~atm})$. Then the appropriate amount of epoxy resin and hardener were weighed out, and degassed separately by using a vacuum oven $\left(103 \mathrm{~Pa}, 50^{\circ} \mathrm{C}\right)$ for $15 \mathrm{~min}$. The ratio of epoxy and resin is 1000:344. The resin was then injected into the small glass vessel contains nanoparticles and mixed. The mixture was then sonicated, with cooling breaks in order to avoid too much heat build-up, which may initiate curing or decompose the resin. Then the hardener was added and the compound mixed at $600 \mathrm{rpm}$ and $50^{\circ} \mathrm{C}$ for $15 \mathrm{~min}$. The composite was degassed at $103 \mathrm{~Pa}$ and $50{ }^{\circ} \mathrm{C}$ for $30 \mathrm{~min}$. After being degassed, the mixture was subsequently cast by nitrogen pressure into a steel mould to produce thin films, then the samples were cured at $120{ }^{\circ} \mathrm{C}$. All the samples were controlled in the vacuum oven $\left(103 \mathrm{~Pa}, 60^{\circ} \mathrm{C}\right)$ for $72 \mathrm{~h}$ and then stored in the vacuum desiccator with dried silica gel at $20^{\circ} \mathrm{C}$. The samples were coded as EPS and EPST, in which $\mathrm{S}$ for untreated nano-silica composites and ST for treated silica. For example, EPS05 represents epoxy nanocomposites filled with $0.5 \mathrm{wt} \%$ untreated silica.

\subsection{EXPERIMENT SET-UP}

EVO 50 scanning electron microscopy (SEM) was used to characterise the morphology of epoxy and its nanocomposites. The gun voltage was set to $15 \mathrm{kV}$ with working distance of 7-12 mm. All samples have been coated with gold before test by the Emitech K550X sputter coater at $25 \mathrm{~mA}$ for $3 \mathrm{~min}$ for each sample.

The breakdown test was performed based on ASTM standard D149-87. The breakdown strengths of epoxy samples at $50 \mathrm{~Hz}$ were measured with a ramping rate of $50 \mathrm{~V} \cdot \mathrm{s}^{-1}$. DC breakdown strength was measured with a ramp rate of $100 \mathrm{~V} \cdot \mathrm{s}^{-1}$ with positive polarity. The thickness of tested samples is $0.085 \pm 0.01 \mathrm{~mm}$. Test points selected were uniformly distributed on each sample and a total of 25 breakdown data are collected for each type of samples. Sample films were immersed in silicone oil between two steel ball bearings with a diameter of $0.635 \mathrm{~cm}$ at each side. It has been found that about 15 breakdowns will cause pitting on electrodes. Therefore, during the test, the ball bearings were changed after every 10 breakdown tests. Finally, the data were analysed by Weibull 7++ Software.

\section{QUANTIFICATION METHOD FOR SEM CHARACTERIZATION}

This section is going to introduce some quantification method and then use the combined method to analyse the existing SEM images in order to relate dispersion and distribution of nanofillers in nanocomposites with their dielectric properties.

\subsection{EQUIVALENT DIAMETER AND HISTOGRAM WEIGHTING METHOD}

Based on the definition discussed before, the dispersion of particles is the first factor to be considered when we estimate the mixing state of a nanocomposite. As the degree of dispersion is only related to the particle count of aggregates 
and particles [12] and most of particles observed by SEM is not the primary ones and exist as aggregates, in this research, equivalent diameter is chosen to estimate the dispersion degree as shown in Fig. 1 and could be calculated by Equation 1 as below:

$$
D_{E}=2 \cdot \sqrt{A_{a} / \pi}
$$

where $D_{E}$ is the equivalent diameter and $A_{a}$ is measured area of aggregates.

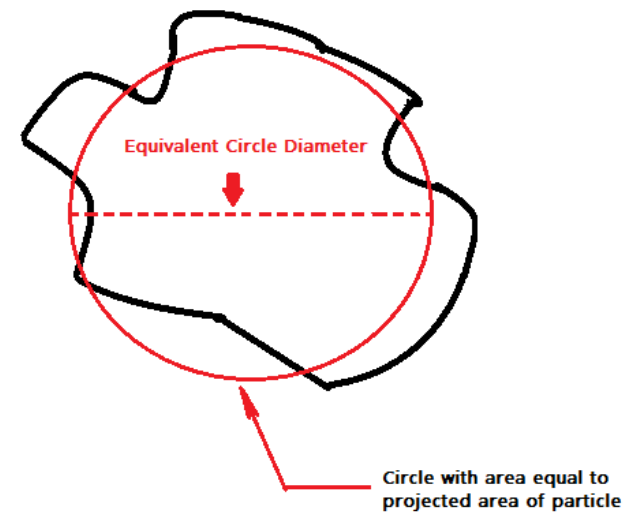

Figure 1. Illustration of equivalent diameter of particles/aggregates.

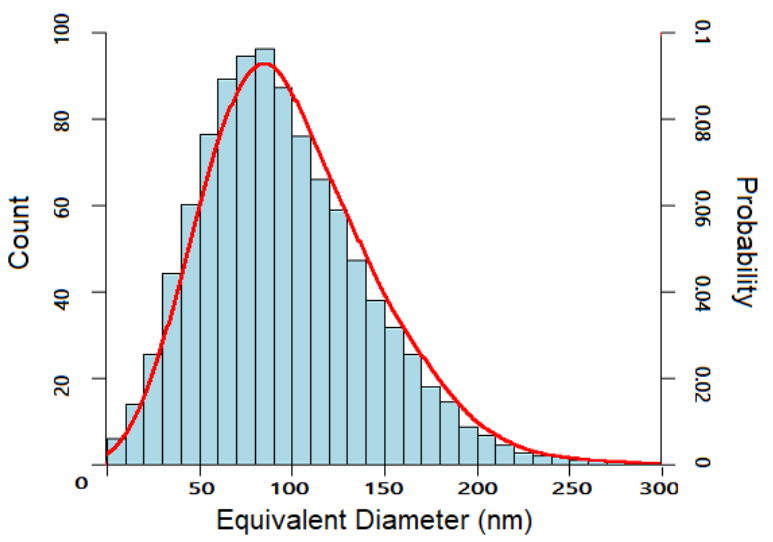

Figure 2. Histogram to generate weighted average of equivalent diameter.

The arithmetic mean of equivalent diameters is not precise enough for use as a measure of dispersion due to the variation of particle numbers in specific areas. Improved dispersion metrics can be obtained by considering the probability distribution of the equivalent diameter data which is to generate some squares as bins and then to collect numbers of into each bin based on the fulfilment condition of each square. The area of each square is proportional to the count numbers and a histogram can be generated according to the existing data as shown in Fig. 2. Moreover, a red fitting curve is also given to reflect the occurrence probability of each condition shown as size of each square in the histogram. The weighted average of equivalent diameter seems to be a good metric of the dispersion degree of particles in nanocomposites [15]. The standard deviation or the weighted standard deviation of the equivalent diameter data can also be used to evaluate the dispersion degree [12].

\subsection{NEAREST NEIGHBOR DISTANCE (NND) METHOD}

The nearest neighbour distance (NND) method is based on a calculation of the distance between the centre of mass of each particle/aggregate and its nearest neighbour; it has been widely used by many researchers [16]. For an arbitrary particle/aggregate, the distance between this one and others can be calculated and sorted descendingly. In the resulting distances, the $1^{\text {st }}$ nearest neighbor distance is usually used to estimate the distribution of particles/aggregates. Moreover, according to the discussion on the dispersion of particles, the $1^{\text {st }} \mathrm{NND}$ data is best interpreted via Histogram by evaluating the weighted parameters.

\subsection{QUADRAT BASED METHOD}

The Quadrat test of randomness is a widely used method to investigate the distribution of points in a specific area [17] and can calculate the deviation of particles/aggregates in different parts of polymer nanocomposites. The principle of the method is first to divide the area into equal quadrats of small size based on the scale of particle/aggregate [11]. Then the number of particles in each quadrat is collected and an index called skewness is calculated using Equation 2 [17]:

$$
\text { skewness }=\frac{N}{(N-1)(N-2)} \sum_{i}^{N}\left(\frac{x_{i}-\bar{x}}{\sigma}\right)^{3}
$$

where $N$ is total number of particles, $x_{i}$ is the number of particle/-s in $i^{\text {th }}$ quadrat, $\bar{x}$ and $\sigma$ is average and standard deviation of $x_{i}$. As particles/aggregates would sometimes form large agglomerations of particles/aggregates and leave most of quadrats empty, only using $1^{\text {st }}$ NND is not accurate enough. The non-zero skewness means asymmetry and higher value implies poorer distribution of particles/aggregates.

\subsection{ORIGINAL IMAGES AND PROCESSING}

The SEM images of EPS and EPST samples in 3wt\% and $5 \mathrm{wt} \%$ are shown in Fig. 3. It can be observed that the number of particles visible in both the EPS and EPST samples increases with filler concentration. From EPS3 and EPST3 samples, obvious agglomerations of particles or aggregates have appeared, and it seems that the fillers have had a significant impact on the structure of the matrix, especially in EPS samples. Comparing EPS and EPST samples, it is easy to note that surface treatment acts to reduce agglomeration effects of nanoparticles on the polymer matrix, this is especially clear for high filler concentrations of $\mathrm{SiO}_{2}$ samples. EPST3 and EPST5 samples seem to show relatively better dispersion and distribution.

The analysis above is informative but is not precise enough, therefore, this section introduces image processing methods for more quantitative analysis. The EPS and EPST samples of 1, 3, $5 \mathrm{wt} \%$ were chosen for quantitative analysis with the EPS3 sample shown as an example in Fig. 4 (Normally at least 2 SEM images were processed for each kind of sample and the numbers of nanoparticles per image of $0.5 \mathrm{wt} \%$ samples are not notable enough to be processed). In order to get sufficient spatial resolution to resolve 

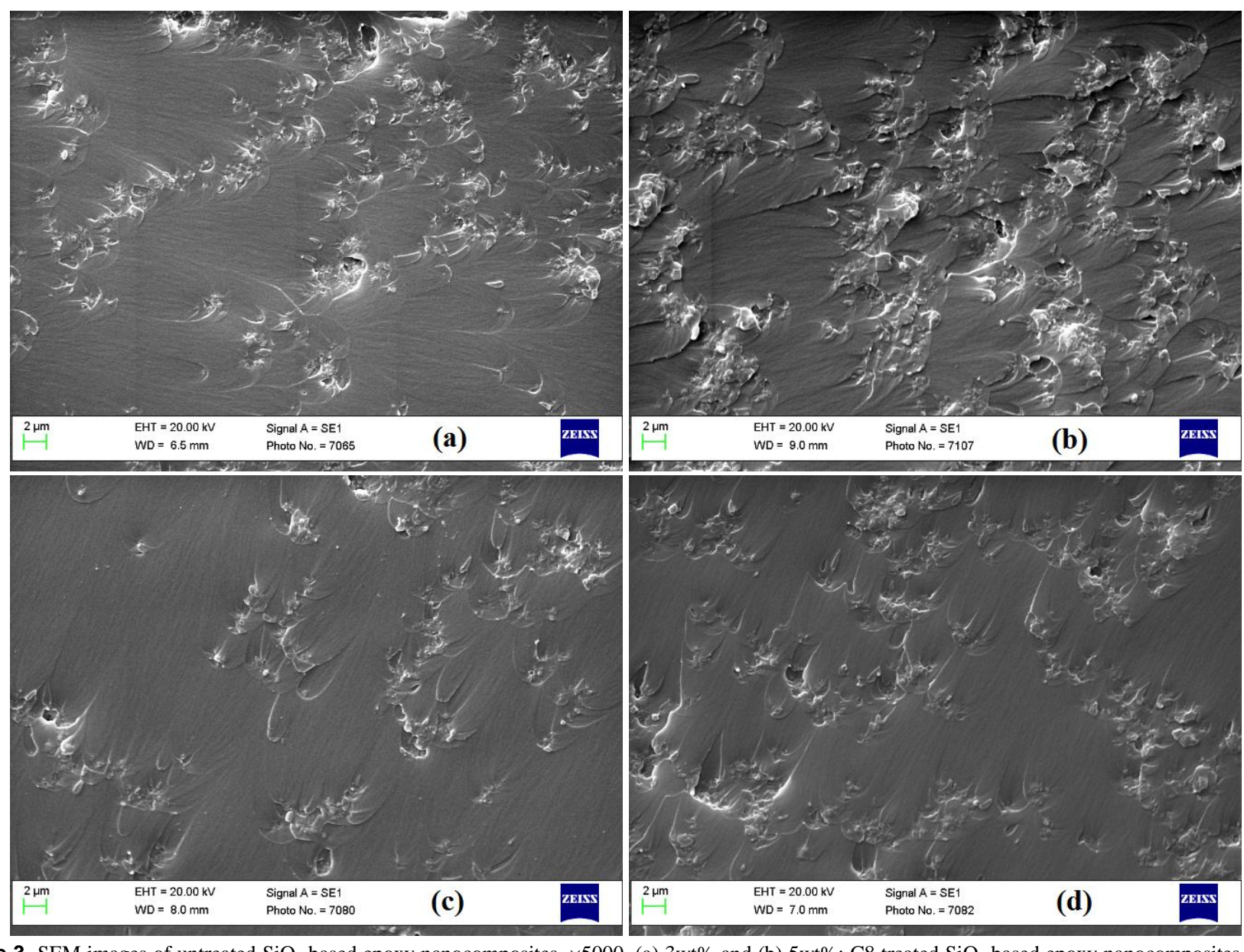

Figure 3. SEM images of untreated $\mathrm{SiO}_{2}$ based epoxy nanocomposites, $\times 5000$, (a) $3 \mathrm{wt} \%$ and (b) $5 \mathrm{wt} \%$; $\mathrm{C} 8$ treated $\mathrm{SiO}_{2}$ based epoxy nanocomposites, $\times 5000$, (c) $3 \mathrm{wt} \%$ and (d) $5 \mathrm{wt} \%$.

individual nano-particles (10-20 nm), SEM images recorded at a magnification of $\times 15000$ were used (16-17 nm spatial resolution). Image ${ }^{\circledR}$ software is used to digitalize images and generate data such as the coordinates of mass centers and the areas of each particle/aggregate (example binary images are shown in Fig. 4 (c) and (d)). Particles that could be clearly recognised were selected and their circularity was calculated based on 50 sampled particles/aggregates; it was found that this ranged between 0.3 and 0.6. Thus, this method could also be used in other polymer nanocomposites filled with sphere/like particles. Features in the binary images can then be filtered based on their circularity so that structures of the matrix, like cracks shown in Fig. 4 (b) are ignored. In this manner particles/aggregates within an image can be identified (blue circled) as shown in Fig. 4 (e) and (f).

For comparison, histograms and occurrence probability curves of measured particle/aggregate size and $1^{\text {st }} \mathrm{NND}$ of EPS3 and EPST3 are shown in Fig. 5 and 6. It can be noticed that EPST3 samples have smaller particles' size and larger inter-particle distance when compared to EPS3, which means EPST3 has better dispersion and distribution of particles in bulk. From the perspective of quantitative data, a combined method of weighted average equivalent diameters (WAED) and deviations, $1^{\text {st }}$ NND and skewness of particles are applied to help analysis dispersion and distribution of particles/aggregates. For skewness, a quadrat size of 32000 $\mathrm{nm}^{2}$ is chosen [11]. These data are shown in Table 1.

For EPS samples: In the case of EPS3 and EPS5 WAED and its deviation increase with the growth of filler loadings. Smaller WAED and deviation means smaller aggregates and better dispersion of particles [12]. The lower value in EPS3 than EPS5 could be due to the probe sonication, since there are fewer particles but they are subjected to the same amount of sonication energy. However, EPS1 samples show a much larger value in WAED. This is probably because there is a much lower number of particles with many of them being agglomerated together. This is also reflected in the larger value in deviation of WAED (165.60) and skewness of 12.66 for the EPS1 samples. The weighted $1^{\text {st }} \mathrm{NND}$ and the skewness in EPS samples both decrease as the filler loading is increased. For a given filler loading lower weighted $1^{\text {st }}$ NND and lower skewness together indicates better dispersion and more even distribution. However, the relatively low value of weighted $1^{\text {st }}$ NND for EPS3 could be due to the asymmetry of distributions which leaves many areas blank as shown in Fig. 4 (a) and (b). This asymmetry is also reflected in its relatively large value of skewness (8.05). For the EPST samples, the overall values indicate better dispersion and distribution when compared with EPS samples of the same filler loadings; this is the expected result of effective surface treatment. Moreover, if particles/aggregates are regarded as recombination centers 

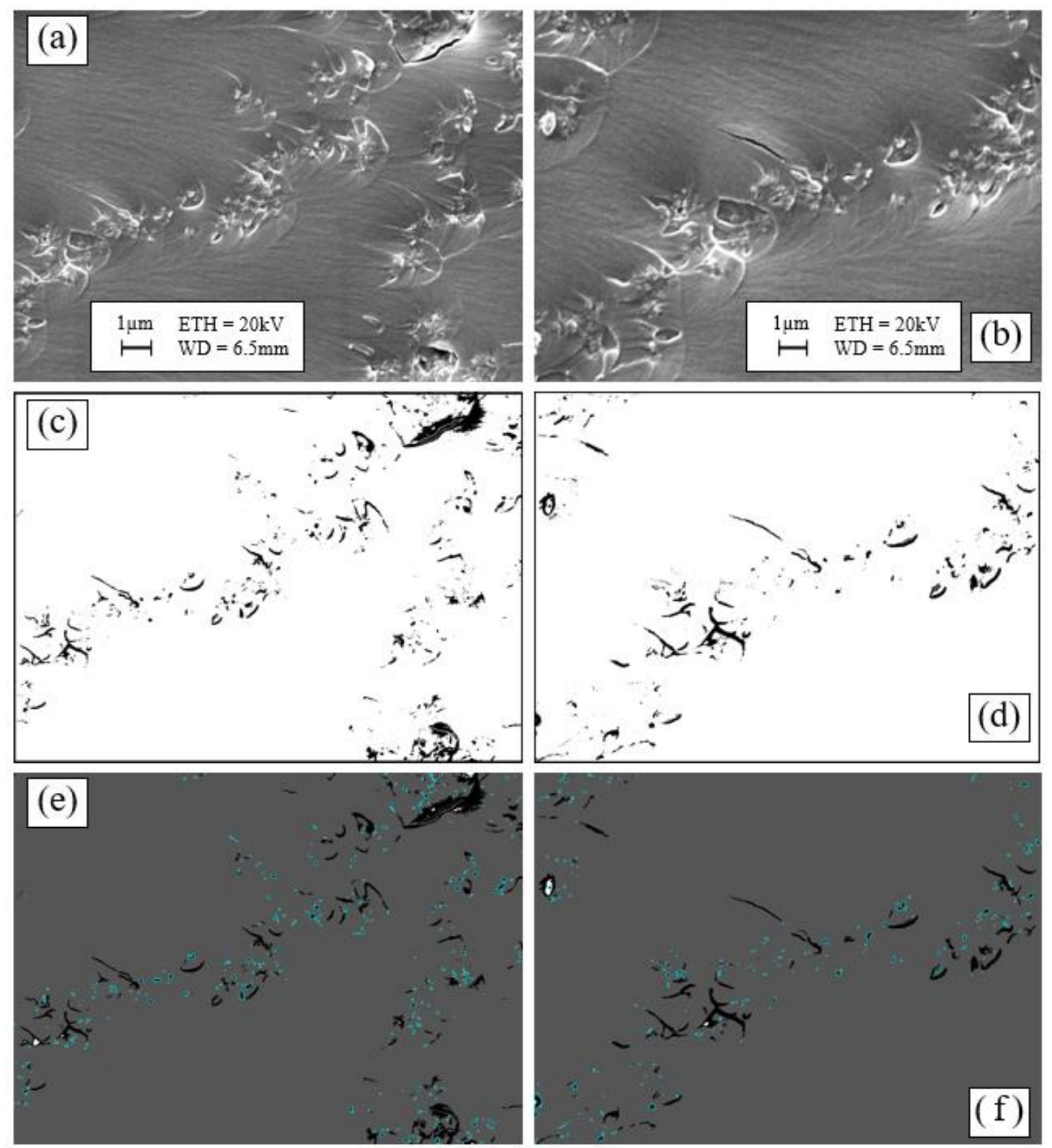

Figure 4. SEM images of untreated $\mathrm{SiO}_{2}$ based epoxy nanocomposites, $3 \mathrm{wt} \%, \times 15000$, (a)(b) Original SEM images; (c)(d) Binary SEM images; (e)(f) Processed SEM images with highlighted particles.

for charge transport [18], then another interesting value, Weighted Average Surface Distance (WASD), can be considered as a method to relate morphology to dielectric properties. Thus, these values are shown for EPS and EPST samples in Table 2.

\section{BREAKDOWN TESTS}

\subsection{AC BREAKDOWN}

The Weibull parameters of AC breakdown in EPS (shown in Fig. 7 (a)) and EPST samples are listed in Table 3 and compared in Fig. 8 (a). It is noticed that the scale parameter of EPS samples decrease with the growth of filler loading concentrations while the EPST samples show the contrary trend. There are two possible reasons why AC breakdown strength in EPS reduces with the growth of filler loading concentrations. First, the overall values are lower than the unfilled samples due to the presence of nanoparticles which act as impurities in the nanocomposites [19] and the consequent enhancement of electric field can be observed around the particles [7]. Second, spherical nanoparticles sometimes can play a role as recombination centres [18], and with the growth of filler loadings, inter-particle/aggregate distances (WASD) (see in Table 2), or in other words these "recombination centres", will decrease, as shown in the SEM analysis results. Thus, transport of charges becomes easier from one centre to another. This phenomenon will result in lowing the percolation threshold of nanocomposites [20] and lead to the final reduction in BD strength when compared to pure epoxy samples. However, according to research by Zhou et al. [21], AC breakdown initiated, most of the time, at layers near the surfaces of film samples rather than striking 
Table 1. Quantitative Data of SEM Images, EPS and EPST Samples in 1, 3, 5 wt\%.

\begin{tabular}{|c|c|c|c|c|}
\hline Sample Code & $\begin{array}{c}\text { Weighted Average Equivalent } \\
\text { Diameter } \\
(\mathrm{nm})\end{array}$ & $\begin{array}{c}\text { Weighted Average Equivalent } \\
\text { Diameter Deviation }\end{array}$ & $\begin{array}{l}\text { Weighted 1st NND } \\
(\mathrm{nm})\end{array}$ & Skewness \\
\hline EPS1 & 169.39 & 165.60 & 291.11 & 12.66 \\
\hline EPS3 & 93.58 & 68.54 & 218.48 & 8.05 \\
\hline EPS5 & 100.79 & 70.22 & 177.60 & 6.25 \\
\hline EPST1 & 160.27 & 120.65 & 353.12 & 11.69 \\
\hline EPST3 & 75.12 & 51.14 & 277.10 & 6.44 \\
\hline EPST5 & 89.55 & 66.66 & 245.16 & 5.71 \\
\hline
\end{tabular}

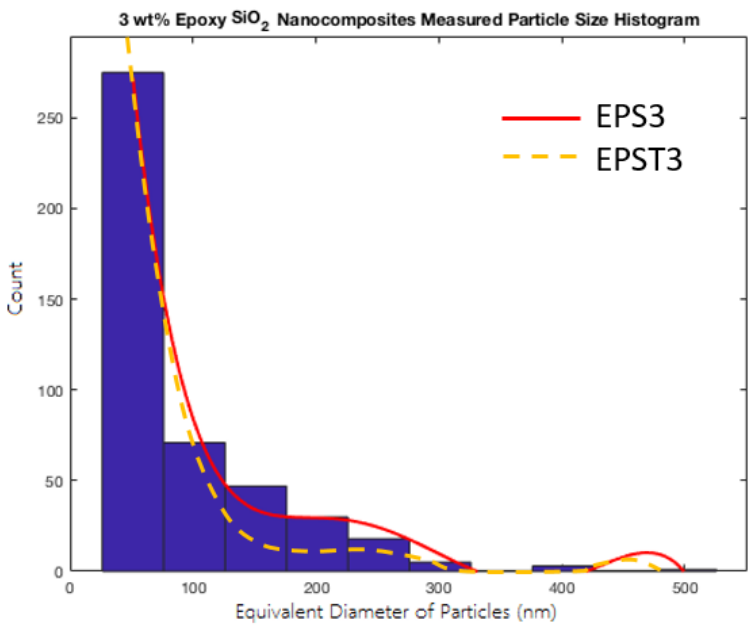

Figure 5. Histogram of measured particle/aggregate size of EPS3 (occurrence probability curve of EPST3 shown as a dashed line).

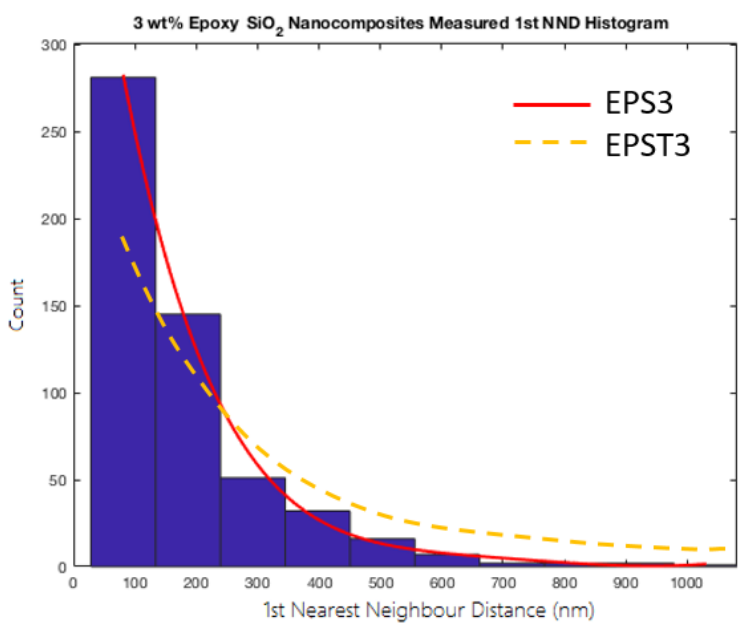

Figure 6. Histogram of measured 1st Nearest Neighbor Distance of EPS3 (occurrence probability curve of EPST3 shown as a dashed line).

Table 2. Weighted Average Surface Distance of EPS and EPST in 1, 3, $5 \mathrm{wt} \%$.

\begin{tabular}{c|c}
\hline Sample Code & $\begin{array}{c}\text { Weighted Average Surface } \\
\text { Distance } \\
\text { (nm) }\end{array}$ \\
\hline EPS1 & 180.43 \\
EPS3 & 155.27 \\
EPS5 & 121.59 \\
EPST1 & 257.35 \\
EPST3 & 200.08 \\
EPST5 & 157.97 \\
\hline
\end{tabular}

through the whole bulk. Thus, the observed reduction in inter-particle/aggregate distances with growth of filler loadings based on the study of the whole material cannot be directly related to the $\mathrm{AC} \mathrm{BD}$ behavior of $\mathrm{SiO}_{2}$ particles based epoxy nanocomposites but the local dispersion and distribution in the BD regions instead. This should be the reason why there is no obvious relationship between $\mathrm{AC} \mathrm{BD}$ strength and filler loading concentrations which can be observed in Fig. 7 (a) and Fig.8 (a).

In EPST samples, there shows a small increase with growth of filler loadings, which might be due to the presence of additional traps resulting from the functionalised surface at interphase [22, 23], and the density of traps at interphase would increase with loadings and then impairs the mobility of charge carriers. However, according to the results, dispersion and distribution of particles may not have obvious effect on AC breakdown strength of nanocomposites which has also been reported in [24]. The main reason why EPST shows worse AC BD than EPS samples should be the impurities introduced by surface treatment and provide more charge carries, which was also reported in [14]. The higher BD strength in samples of $5 \mathrm{wt} \%$ is due to the better dispersion and distribution of particles and resultant smaller

(a) EPS

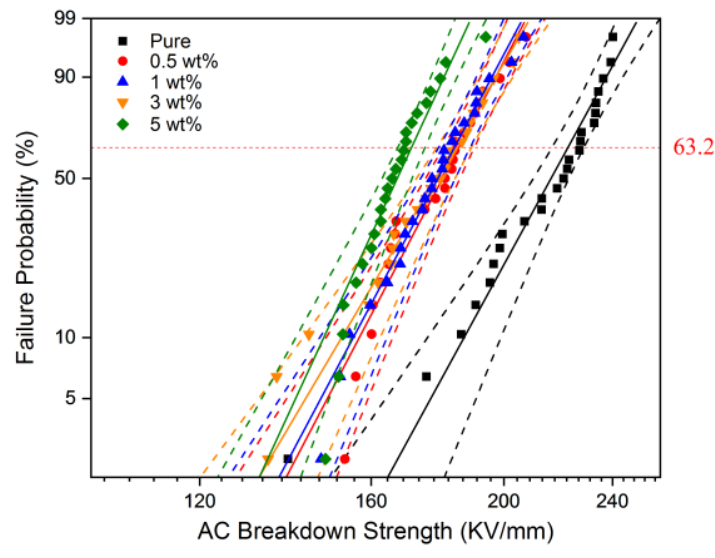

(b) EPS

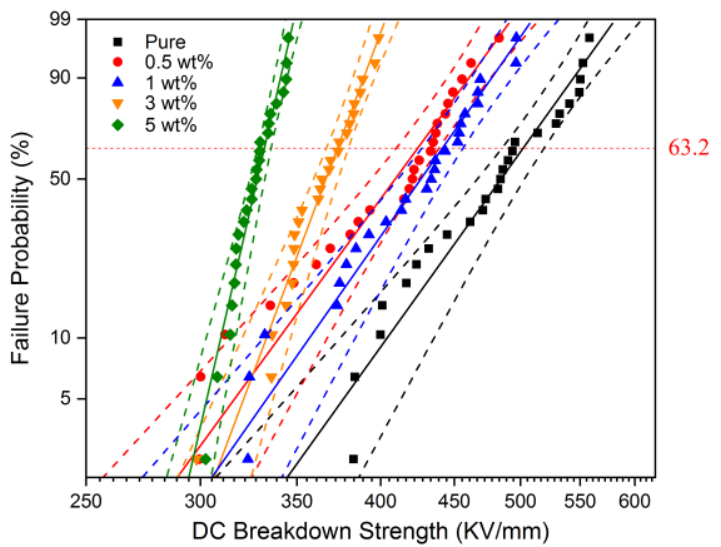

Figure 7. Weibull plot of (a) AC and (b) DC BD strength of untreated nano $\mathrm{SiO}_{2}$ filled epoxy composites in different loading concentrations. 
Table 3. Weibull Parameters from MLE for Samples under AC and DC Breakdown Test

\begin{tabular}{|c|c|c|c|c|}
\hline \multirow[b]{2}{*}{ Sample Code } & \multicolumn{2}{|c|}{$\mathrm{AC}$} & \multicolumn{2}{|c|}{$\mathrm{DC}$} \\
\hline & $\begin{array}{c}\text { Scale } \\
\text { Parameter }(\eta) \\
\left(\mathrm{kV} \cdot \mathrm{mm}^{-1}\right)\end{array}$ & $\begin{array}{l}\text { Shape Parameter } \\
(\beta)\end{array}$ & $\begin{array}{c}\text { Scale } \\
\text { Parameter }(\eta) \\
\left(\mathrm{kV} \cdot \mathrm{mm}^{-1}\right)\end{array}$ & $\begin{array}{c}\text { Shape Parameter } \\
(\beta)\end{array}$ \\
\hline EP0 & $222.7 \pm 6.1$ & 12.9 & $502.1 \pm 17.3$ & 10.4 \\
\hline EPS05 & $185.4 \pm 4.9$ & 13.5 & $424.3 \pm 10.3$ & 10.2 \\
\hline EPS1 & $184.0 \pm 5.0$ & 13.2 & $441.4 \pm 7.6$ & 10.6 \\
\hline EPS3 & $183.9 \pm 5.5$ & 11.9 & $373.3 \pm 7.0$ & 20.0 \\
\hline EPS5 & $171.2 \pm 4.0$ & 15.3 & $332.2 \pm 5.0$ & 32.4 \\
\hline EPST05 & $165.9 \pm 5.8$ & 10.2 & $402.7 \pm 14.9$ & 14.0 \\
\hline EPST1 & $170.6 \pm 2.5$ & 24.1 & $436.2 \pm 14.8$ & 20.5 \\
\hline EPST3 & $166.9 \pm 3.4$ & 17.4 & $410.7 \pm 6.7$ & 21.0 \\
\hline EPST5 & $182.0 \pm 4.1$ & 15.9 & $366.2 \pm 3.7$ & 26.2 \\
\hline
\end{tabular}

aggregates as shown in SEM results to which the surface treatment contributed. This means the surface treatment can help $\mathrm{SiO}_{2}$ particles, at least, disperse better and improve the $\mathrm{AC}$ breakdown strength in samples of higher filler loading concentration at local areas of BD.

(a)

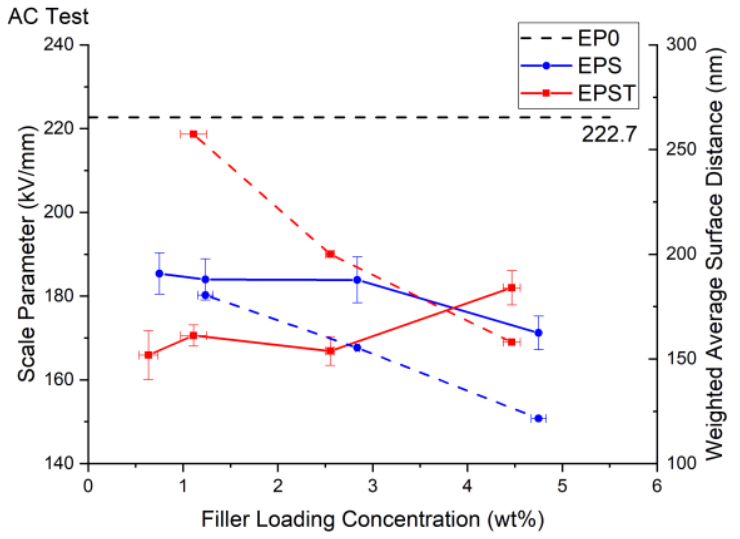

(b)

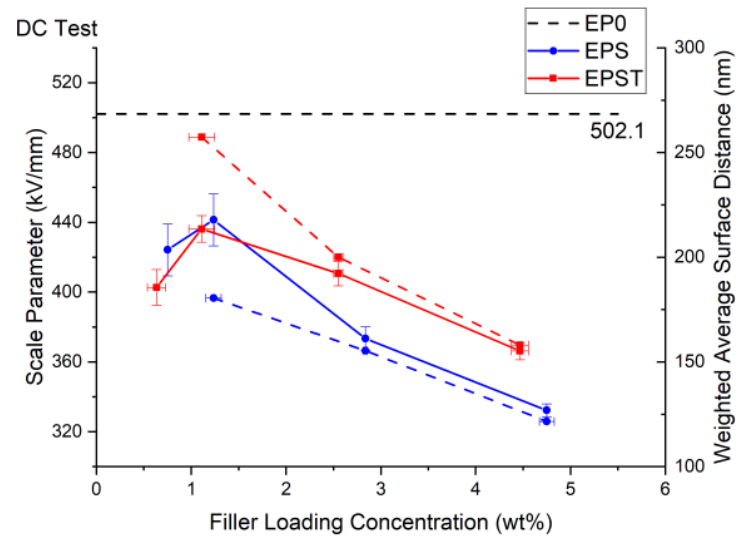

Figure 8. Comparisons of AC and DC BD strength (solid) and weighted average surface distances (dashed) of EPS and EPST in different loadings concentrations.

\subsection{BREAKDOWN}

The Weibull parameters of DC breakdown in EPS (shown in Fig. 7 (b)) and EPST samples are listed in Table 3 and compared in Fig. 8 (b). The results of DC breakdown in EPS and EPST samples show very similar trends, The higher DC BD of EPS1 and EPST1 than EPS05 and EPST05 respectively should be mainly caused by that the presence of more nano- $\mathrm{SiO}_{2}$ particles introduces more traps, especially ones near surface acting as a barrier and thus hindering the injection of charges, consequently increases breakdown strength. However, in higher loadings, the enhancement of electric field intensity caused by aggregates and agglomerations lead to the worse performance of $\mathrm{BD}$ strength. Second, unlike the observations in AC BD, DC BD strengths decrease obviously while filler loadings increase and are highly related to the WASD as shown in Fig.8 (b) which is also observed in the results of EPST samples. The growth of loadings will lead to a decrease of interparticle/aggregate and percolation of charge carriers in the whole bulk become easier with increasing loadings. In other words, the mobility of charge has been increased [24]. Under $0.5 \mathrm{wt} \%$ and $1 \mathrm{wt} \%$, the BD strengths of EPS and EPST are very close while the later one is a bit lower. As the numbers of nanoparticles per image of $0.5 \mathrm{wt} \%$ samples are not notable enough to be processed, it is hard to distinguish in EPS and EPST which has a better dispersion of nanoparticles. But at least, it could be stated that the lower value in EPST should be influenced by the impurities introduced by surface treatment [14]. This could be further supported by our previous research [25] in which EPST05 has higher DC conductivity than that of EPS05. Differently, under higher filler loading concentrations, dispersion and distribution of particles/aggregates become more dominant while influencing the DC BD strength of epoxy nanocomposites based on the comparison in Fig. 8 (b), and thus DC BD strengths of EPST are higher.

\section{CONCLUSIONS}

In this paper, first, a combined quantification method to estimate the dispersion and distribution of spherical/-like particles/aggregates in polymer nanocomposites has been developed. Based on the proposed quantification method, epoxy nanocomposite with surface treated $\mathrm{SiO}_{2}$ shows overall better dispersion and distribution of particles/aggregates than those with untreated ones.

In AC breakdown tests, agglomerations of particles/aggregates, observed in SEM results, led to the reduction of breakdown strength. However, dispersion and distribution of particles had limited influence on that. In DC breakdown tests, the growth of filler loadings led to a decrease of inter-particle/aggregate distances. Thus the percolation of charge carriers in the whole bulk could become easier and resulted in the reduction of DC BD strength, where the dispersion and distribution of particles/aggregates provide the support qualitatively. 


\section{REFERENCES}

[1] R. Roy, S. Komarneni, and D. Roy, "Multi-phasic ceramic composites made by sol-gel technique," MRS Online Proceedings Library Archive, vol. 32, 1984.

[2] A. P. Kumar, D. Depan, N. S. Tomer, and R. P. Singh, "Nanoscale particles for polymer degradation and stabilization - trends and future perspectives," Progress in polymer science, vol. 34, no. 6, pp. 479-515, 2009.

[3] C. Zhang and G. C. Stevens, "The dielectric behaviour of the interface in polymer nanocomposites," IEEE Int. Confer. in Solid Dielectrics (ICSD), 2007, pp. 423-427.

[4] T. Lewis, "Interfaces are the dominant feature of dielectrics at the nanometric level," IEEE Trans. Dielectr. Electr. Insul., vol. 11, no. 5 , pp. 739-753, 2004.

[5] T. Imai, F. Sawa, T. Ozaki, Y. Inoue, T. Shimizu, and T. Tanaka, "Comparison of insulation breakdown properties of epoxy nanocomposites under homogeneous and divergent electric fields," Annu. Rep. Conf. Electr. Insul. Dielect. Phenom. (CEIDP), 2006, pp. 306-309.

[6] S. Singha and M. J. Thomas, "Dielectric properties of epoxy nanocomposites," IEEE Trans. Dielectr. Electr. Insul., vol. 15, no. 1, 2008.

[7] J. Nelson and Y. Hu, "Nanocomposite dielectrics-properties and implications," Journal of Physics D: Applied Physics, vol. 38, no. 2, p. $213,2005$.

[8] Q. Wang and G. Chen, "Effect of pre-treatment of nanofillers on the dielectric properties of epoxy nanocomposites," IEEE Trans. Dielectr. Electr. Insul., vol. 21, no. 4, pp. 1809-1816, 2014

[9] M. Roy, J. K. Nelson, R. K. MacCrone, L. S. Schadler, C. W. Reed, and R. Keefe, "Polymer nanocomposite dielectrics-the role of the interface," IEEE Trans. Dielectr. Electr. Insul., vol. 12, no. 4, pp. 629-643, 2005.

[10]I. L. Hosier, M. Praeger, A. F. Holt, A. S. Vaughan, and S. G. Swingler, "On the effect of functionalizer chain length and water content in polyethylene/silica nanocomposites: Part I-Dielectric properties and breakdown strength," IEEE Trans. Dielectr. Electr. Insul., vol. 24, no. 3, pp. 1698-1707, 2017.

[11]D. Kim, J. S. Lee, C. M. Barry, and J. L. Mead, "Microscopic measurement of the degree of mixing for nanoparticles in polymer nanocomposites by TEM images," Microscopy research and technique, vol. 70, no. 6, pp. 539-546, 2007.

[12] L. Hui, R. C. Smith, X. Wang, J. K. Nelson, and L. S. Schadler, "Quantification of Particulate Mixing in Nanocomposites," Annu. Rep. Conf. Electr. Insul. Dielect. Phenom. (CEIDP), 2008, pp. 317-320.

[13]L. Hui, L. S. Schadler, and J. K. Nelson, "The influence of moisture on the electrical properties of crosslinked polyethylene/silica nanocomposites," IEEE Trans. Dielectr. Electr. Insul., vol. 20, no. 2, pp. 641-653, 2013

[14]K. Y. Lau, "Structure and electrical properties of silica-based polyethylene nanocomposites," $\mathrm{PhD}$ thesis, University of Southampton, 2013.

[15]Y. Zhou, J. Hu, X. Chen, F. Yu, and J. He, "Thermoplastic polypropylene/aluminum nitride nanocomposites with enhanced thermal conductivity and low dielectric loss," IEEE Trans. Dielectr. Electr. Insul., vol. 23, no. 5, pp. 2768-2776, 2016

[16] J. W. Leggoe, "Nth-nearest neighbor statistics for analysis of particle distribution data derived from micrographs," Scripta materialia, vol. 53, no. 11 , pp. 1263-1268, 2005.

[17]P. J. Diggle, "Statistical analysis of spatial point processes," Academic, London, 1983

[18] T. Lewis, "Polyethylene under electrical stress," IEEE Trans. Dielectr. Electr. Insul., vol. 9, no. 5, pp. 717-729, 2002.

[19] S. Kang, S. I. Hong, C. R. Choe, M. Park, S. Rim, and J. Kim, "Preparation and characterization of epoxy composites filled with functionalized nanosilica particles obtained via sol-gel process," Polymer, vol. 42, no. 3, pp. 879-887, 2001.

[20]M. F. Fréchette et al., "Nanodielectrics: A "universal" panacea for solving all electrical insulation problems?," IEEE Int. Confer. in Solid Dielectrics (ICSD), 2010, pp. 1-3.

[21]C. Zhou and G. Chen, "Space charge and AC electrical breakdown strength in polyethylene," IEEE Trans. Dielectr. Electr. Insul., vol. 24, no. 1, pp. 559-566, 2017.
[22]S. Li, D. Min, W. Wang, and G. Chen, "Linking traps to dielectric breakdown through charge dynamics for polymer nanocomposites," IEEE Trans. Dielectr. Electr. Insul., vol. 23, no. 5, pp. 2777-2785, 2016.

[23] K. Lau, A. Vaughan, G. Chen, I. Hosier, and A. Holt, "On the dielectric response of silica-based polyethylene nanocomposites," Journal of Physics D: Applied Physics, vol. 46, no. 9, p. 095303, 2013.

[24] K. Lau, A. Vaughan, G. Chen, I. Hosier, A. Holt, and K. Y. Ching, "On the space charge and DC breakdown behavior of polyethylene/silica nanocomposites," IEEE Trans. Dielectr. Electr. Insul., vol. 21, no. 1, pp. 340-351, 2014.

[25]D. Qiang et al., "The Effect of Filler Loading Ratios and Moisture on DC Conductivity and Space Charge Behavior of $\mathrm{SiO}_{2}$ and hBN Filled Epoxy Nanocomposites," Journal of Physics D: Applied Physics, 2019, https://doi.org/10.1088/1361-6463/ab2d5b.

\section{BIOGRAPHIES}

Dayuan Qiang was born in Lanzhou, China in 1989. He received the B.Sc. degree from Northwest A\&F University, Shaanxi Province, China in 2012. He obtained his Ph.D. degree in the University of Southampton in 2018 and now works as a postdoctral research at the University of Exeter. His research area are nanodielectrics and nanomaterials for additive manufacturing.

Yan Wang was born in Lanzhou, China in 1990. $\mathrm{He}$ received the B.Sc. degree from Liverpool University, China in 2013. He obtained his Ph.D. degree in the University of Southampton in 2018. His research area is the nano dielectrics based on polyethylene.

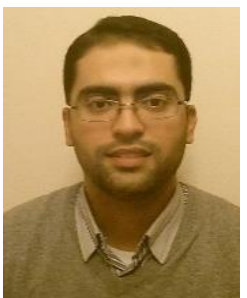

Fuad N. Alhabill received his BSc (with distinction) in 2008 from the Islamic University of Gaza, Palestine, where he worked as a teaching assistant and lecturer until 2011. He was awarded Said Foundation scholarship and received MSc (with distinction) from the University of Southampton, UK, in 2014. In 2017 he has completed a Ph.D. in high voltage power engineering at University of Southampton, UK

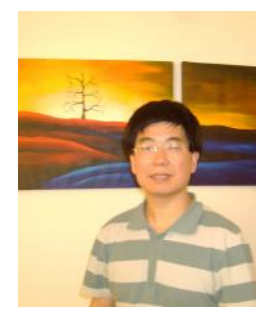

George Chen was born in China in 1961. He received the B.Eng. (1983) and M.Sc. (1986) degrees in electrical engineering from Xi'an Jiao tong University, China. After he obtained the Ph.D. degree (1990) from the University of Strathclyde, UK, he joined the University of Southampton as postdoctoral research fellow and became a senior research fellow subsequently. In 1997 he was appointed as a research lecturer and promoted to a Reader in 2002. He is now the professor of high voltage engineering at the University of Southampton and a visiting professor of Xi'an Jiao tong University.

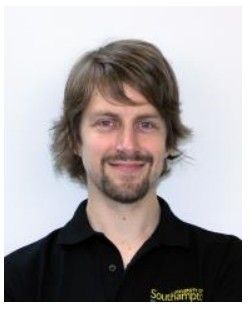

Thomas Andritsch was born in Innsbruck, Austria in 1980. He received the Dipl.-Ing. Degree in electrical engineering from Graz University of Technology in 2006 and his PhD in the same field from Delft University of Technology in 2010. He is currently working at the University of Southampton on advanced and nanomaterials for high voltage application. Thomas is a member of the DEIS technical committee on nanodielectrics and the IEEE working group on nanodielectrics, developing recommended practices for nanocomposite synthesis and preparation. 